\title{
Synthesis, Structural Characterization and Theoretical Studies of Gold(I) and Gold(I)-Gold(III) Thiolate Complexes: Quenching of Gold(I) Thiolate Luminescence.
}

\section{Manuel Bardají, ${ }^{*, a, b, \dagger}$ Maria José Calhorda, ${ }^{c}$ Paulo J. Costa, ${ }^{c}$ Peter G. Jones, ${ }^{\text {d }}$ Antonio Laguna, ${ }^{*, b}$ M. Reyes Pérez ${ }^{\mathrm{b}}$ and M. D. Villacampa.}

a Química Inorgánica, Facultad de Ciencias, Universidad de Valladolid, E-47005 Valladolid, Spain.

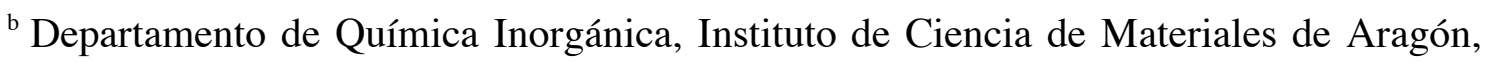
Universidad de Zaragoza-CSIC, E-50009 Zaragoza, Spain.

${ }^{\text {c }}$ Departamento de Química e Bioquímica, Faculdade de Ciências, Universidade de Lisboa, Campo Grande 1749-016 Lisboa, and ITQB, Av. da República, EAN, Apart. 127, 2781-901, Oeiras, Portugal.

d Institut für Anorganische und Analytische Chemie der Technischen Universität, Postfach 3329, D-38023 Braunschweig, Germany.

Table SM1. Composition (\%) and energies $(\mathrm{eV})$ of frontier molecular orbitals of models $\mathbf{1 h}$ and $\mathbf{1 m}$ of complex [Au(2-SC $\left.\left.\mathrm{H}_{4} \mathrm{NH}_{2}\right)\left(\mathrm{PPh}_{3}\right)\right](\mathbf{1})(\mathrm{L}=\mathrm{LUMO}, \mathrm{H}=\mathrm{HOMO})$.

\begin{tabular}{|c|c|c|c|c|c|c|c|}
\hline \multirow{2}{*}{\multicolumn{2}{|c|}{ MO }} & \multirow[b]{2}{*}{ Energy $(\mathrm{eV})$} & \multicolumn{4}{|c|}{ Compositions (\%) } & \multirow[b]{2}{*}{ Main components of orbital } \\
\hline & & & $\mathrm{Au}$ & $\mathrm{S}$ & thiolate & phosphine & \\
\hline \multicolumn{8}{|l|}{ 1h } \\
\hline $38 \mathrm{a}$ & H-3 & -6.752 & 43.2 & 20.2 & 53.5 & 1.1 & $\mathrm{Au}\left(\mathrm{s}, \mathrm{d}_{\mathrm{x} 2-\mathrm{y} 2}\right)+\mathrm{S}\left(\mathrm{p}_{\mathrm{x}}\right)$ \\
\hline $39 a$ & $\mathrm{H}-2$ & -5.550 & 13.6 & 30.9 & 81.1 & 1.9 & $S\left(p_{y}, p_{x}\right)+A u(s)$ \\
\hline $40 \mathrm{a}$ & $\mathrm{H}-1$ & -5.089 & 6.4 & 53.4 & 87.6 & 1.9 & $\mathrm{~S}\left(\mathrm{p}_{\mathrm{y}}, \mathrm{p}_{\mathrm{z}}\right)$ \\
\hline $41 \mathrm{a}$ & $\mathbf{H}$ & -4.596 & 3.8 & 17.1 & 88.8 & 0.9 & $\mathrm{~N}\left(\mathrm{p}_{\mathrm{x}}\right)+\mathrm{C}_{\text {thiolate }}\left(\mathrm{p}_{\mathrm{x}}\right)+\mathrm{S}\left(\mathrm{p}_{\mathrm{z}}\right)$ \\
\hline $42 a$ & $\mathbf{L}$ & -1.710 & 20.2 & 2.3 & 7.1 & 71.0 & $P\left(p_{y}, p_{z}\right)+A u\left(p_{z}, p_{y}\right)$ \\
\hline $43 a$ & $\mathrm{~L}+1$ & -1.557 & 30.9 & 3.8 & 11.6 & 57.9 & $\mathrm{P}\left(\mathrm{p}_{\mathrm{z}}, \mathrm{p}_{\mathrm{y}}\right)+\mathrm{Au}(\mathrm{s})$ \\
\hline $44 \mathrm{a}$ & $\mathrm{L}+2$ & -1.507 & 24.5 & 6.0 & 9.0 & 66.1 & $P\left(s, p_{y}, p_{z}\right)+A u(s)$ \\
\hline $45 \mathrm{a}$ & $\mathrm{L}+3$ & -0.748 & 0.1 & 1.9 & 74.6 & 19.3 & $\mathrm{C}_{\text {thiolate }}\left(\mathrm{p}_{\mathrm{x}}\right)$ \\
\hline \multicolumn{8}{|l|}{$1 \mathrm{~m}$} \\
\hline $47 \mathrm{a}$ & H-3 & -6.342 & 66.7 & 5.2 & 23.7 & 8.0 & $\mathrm{Au}\left(\mathrm{s}, \mathrm{d}_{\mathrm{x} 2-\mathrm{y} 2},{ }_{\mathrm{z} 2}\right)$ \\
\hline $48 \mathrm{a}$ & $\mathrm{H}-2$ & -5.238 & 12.0 & 31.0 & 79.9 & 3.1 & $\mathrm{~S}\left(\mathrm{p}_{\mathrm{y}}, \mathrm{p}_{\mathrm{x}}\right)$ \\
\hline $49 a$ & $\mathrm{H}-1$ & -4.767 & 6.6 & 49.6 & 86.1 & 1.8 & $\mathrm{~S}\left(\mathrm{p}_{\mathrm{y}}, \mathrm{p}_{\mathrm{z}}\right)$ \\
\hline $50 a$ & $\mathbf{H}$ & -4.325 & 4.2 & 25.1 & 88.4 & 1.6 & $\mathrm{~N}\left(\mathrm{p}_{\mathrm{x}}\right)+\mathrm{C}_{\text {thiolate }}\left(\mathrm{p}_{\mathrm{x}}\right)+\mathrm{S}\left(\mathrm{p}_{\mathrm{z}}\right)$ \\
\hline $51 \mathbf{a}$ & $\mathbf{L}$ & -1.104 & 25.2 & 2.4 & 15.3 & 53.8 & $P\left(p_{y}, p_{z}\right)+A u\left(p_{y}, p_{z}\right)$ \\
\hline $52 \mathrm{a}$ & $\mathrm{L}+1$ & -0.888 & 23.4 & 2.5 & 18.0 & 55.1 & $P\left(p_{y}, p_{z}\right)+A u\left(p_{y}, p_{z}\right)$ \\
\hline $53 a$ & $\mathrm{~L}+2$ & -0.698 & 15.0 & 4.1 & 24.0 & 59.0 & $\mathrm{P}(\mathrm{s})+\mathrm{Au}(\mathrm{s})+\mathrm{H}_{\mathrm{Me}}(\mathrm{s})$ \\
\hline $54 \mathrm{a}$ & $\mathrm{L}+3$ & -0.574 & 8.8 & 2.7 & 29.4 & 58.8 & $\mathrm{H}_{\mathrm{Me}}(\mathrm{s})+\mathrm{P}\left(\mathrm{p}_{\mathrm{x}}\right)$ \\
\hline
\end{tabular}


Table SM2. Composition $(\%)$ and energies $(\mathrm{eV})$ of frontier molecular orbitals of complex $\left[\mathrm{Au}\left(2-\mathrm{SC}_{6} \mathrm{H}_{4} \mathrm{NH}_{2}\right)\left(\mathrm{PPh}_{3}\right)\right]$ (1) $(\mathrm{L}=\mathrm{LUMO}, \mathrm{H}=\mathrm{HOMO})$.

\begin{tabular}{cccccccc}
\hline & & \multicolumn{5}{c}{ Compositions (\%) } \\
\cline { 4 - 7 } \multicolumn{2}{c}{ MO } & Energy $(\mathrm{eV})$ & $\mathrm{Au}$ & $\mathrm{S}$ & thiolate & phosphine & Main components of orbital \\
\hline $\mathbf{1}$ & & & & & & \\
\hline $80 \mathrm{a}$ & $\mathrm{H}-3$ & -6.211 & 44.5 & 0.2 & 2.6 & 46.8 & $\mathrm{Au}(\mathrm{s}) ; \mathrm{Au}\left(\mathrm{d}_{\mathrm{x} 2-\mathrm{y} 2}\right)$ \\
$81 \mathrm{a}$ & $\mathrm{H}-2$ & -5.233 & 11.0 & 30.2 & 79.2 & 3.6 & $\mathrm{~S}\left(\mathrm{p}_{\mathrm{y}}, \mathrm{p}_{\mathrm{x}}\right)$ \\
$82 \mathrm{a}$ & $\mathrm{H}-1$ & -4.777 & 7.4 & 56.3 & 85.1 & 1.2 & $\mathrm{~S}\left(\mathrm{p}_{\mathrm{y}}, \mathrm{p}_{\mathrm{z}}\right)$ \\
$\mathbf{8 3 a}$ & $\mathbf{H}$ & $\mathbf{- 4 . 3 1 4}$ & $\mathbf{3 . 8}$ & $\mathbf{1 8 . 0}$ & $\mathbf{8 8 . 2}$ & $\mathbf{1 . 5}$ & $\mathrm{N}\left(\mathrm{p}_{\mathrm{x}}\right)+\mathrm{C}_{\left(\mathrm{p}_{\mathrm{x}}\right)+\mathrm{S}\left(\mathrm{p}_{\mathrm{z}}\right)}$ \\
$\mathbf{8 4 a}$ & $\mathbf{L}$ & $\mathbf{- 2 . 3 5 2}$ & $\mathbf{1 . 2}$ & $\mathbf{0 . 1}$ & $\mathbf{0 . 3}$ & $\mathbf{9 2 . 6}$ & $\mathrm{C}_{\mathrm{Ph}}\left(\mathrm{p}_{\mathrm{y}}\right)$ \\
$85 \mathrm{a}$ & $\mathrm{L}+1$ & -2.017 & 3.5 & 0.8 & 1.5 & 89.5 & $\mathrm{C}_{\mathrm{Ph}}\left(\mathrm{p}_{\mathrm{x}}\right)$ \\
$86 \mathrm{a}$ & $\mathrm{L}+2$ & -1.764 & 2.6 & 0.2 & 0.4 & 87.4 & $\mathrm{C}_{\mathrm{Ph}}\left(\mathrm{p}_{\mathrm{y}}\right)$ \\
$87 \mathrm{a}$ & $\mathrm{L}+3$ & -1.718 & 2.9 & 0.7 & 1.3 & 87.9 & $\mathrm{C}_{\mathrm{Ph}}\left(\mathrm{p}_{\mathrm{y}}\right)$ \\
\hline
\end{tabular}

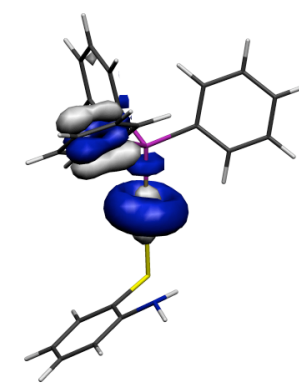

H-3

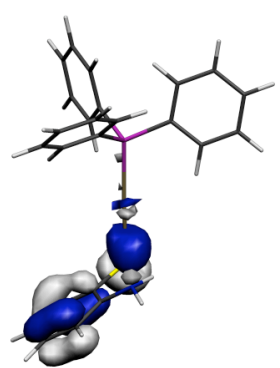

H-2

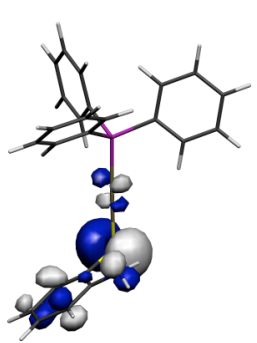

H-1

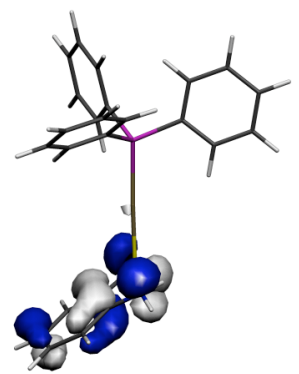

$\mathbf{H}$

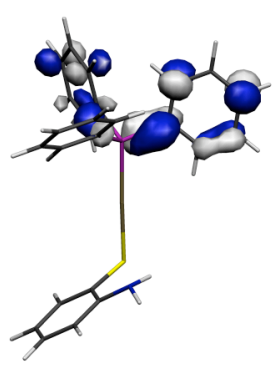

$\mathbf{L}$

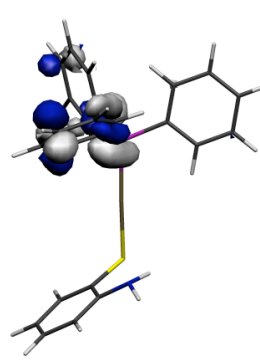

$\mathbf{L}+1$

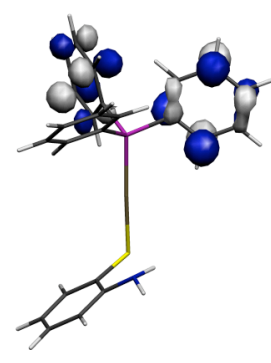

$\mathbf{L}+\mathbf{2}$

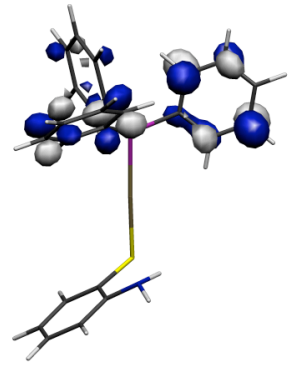

$\mathbf{L}+3$

Figure SM1. Representation of the frontier orbitals of complex $[\mathrm{Au}(2-$ $\left.\left.\mathrm{SC}_{6} \mathrm{H}_{4} \mathrm{NH}_{2}\right)\left(\mathrm{PPh}_{3}\right)\right](\mathbf{1})$ 
Table SM3. Calculated low-energy singlet excitation energies, wavelengths, and oscillator strengths (OS) for models $\mathbf{1 h}$ and $\mathbf{1 m}$ of complex [ $\mathrm{Au}\left(2-\mathrm{SC}_{6} \mathrm{H}_{4} \mathrm{NH}_{2}\right)\left(\mathrm{PPh}_{3}\right)$ ] (1)

\begin{tabular}{|c|c|c|c|c|}
\hline Composition & $\begin{array}{c}\text { Energy } \\
(\mathrm{eV})\end{array}$ & $\begin{array}{c}\text { Wavelength } \\
(\mathrm{nm})\end{array}$ & $\begin{array}{l}\lambda_{\max }^{\mathrm{a}} \\
(\mathrm{nm})\end{array}$ & $\begin{array}{c}\mathrm{OS} \\
\left(\mathrm{x} 10^{3}\right) \\
\end{array}$ \\
\hline \multicolumn{5}{|l|}{ 1h } \\
\hline $60 \%(\mathrm{H} \rightarrow \mathrm{L}+2), 37 \%(\mathrm{H} \rightarrow \mathrm{L}+1)$ & 3.154 & 393 & & 0.046 \\
\hline $64 \%(\mathrm{H}-1 \rightarrow \mathrm{L}+2), 24 \%(\mathrm{H} \rightarrow \mathrm{L}+1)$ & 3.718 & 333 & & 0.044 \\
\hline $75 \%(\mathrm{H}-2 \rightarrow \mathrm{L}), 17 \%(\mathrm{H} \rightarrow \mathrm{L}+3)$ & 4.037 & 307 & & 0.004 \\
\hline $60 \%(\mathrm{H} \rightarrow \mathrm{L}+3), 15 \%(\mathrm{H}-2 \rightarrow \mathrm{L}+1)$ & 4.177 & 297 & & 0.044 \\
\hline $78 \%(\mathrm{H}-2 \rightarrow \mathrm{L}+2)$ & 4.231 & 293 & 311 & 0.178 \\
\hline \multicolumn{5}{|l|}{$1 \mathrm{~m}$} \\
\hline $90 \%(\mathrm{H} \rightarrow \mathrm{L}+1)$ & 3.515 & 353 & & 0.019 \\
\hline $73 \%(\mathrm{H} \rightarrow \mathrm{L}+2), 24 \%(\mathrm{H} \rightarrow \mathrm{L}+3)$ & 3.670 & 338 & & 0.010 \\
\hline $62 \%(H \rightarrow L+3), 17 \%(H \rightarrow L+2)$ & 3.923 & 316 & 311 & 0.102 \\
\hline $80 \%(\mathrm{H}-1 \rightarrow \mathrm{L}+1)$ & 4.004 & 310 & & 0.018 \\
\hline $80 \%(\mathrm{H}-1 \rightarrow \mathrm{L}+2), 16 \%(\mathrm{H}-1 \rightarrow \mathrm{L}+3)$ & 4.104 & 302 & & 0.012 \\
\hline $55 \%(\mathrm{H}-2 \rightarrow \mathrm{L}), 30 \%(\mathrm{H} \rightarrow \mathrm{L}+4)$ & 4.206 & 295 & & 0.029 \\
\hline $\begin{array}{l}38 \%(\mathrm{H} \rightarrow \mathrm{L}+4), 27 \%(\mathrm{H}-2 \rightarrow \mathrm{L}), \\
13 \%(\mathrm{H}-1 \rightarrow \mathrm{L}+3)\end{array}$ & 4.244 & 292 & & 0.015 \\
\hline $58 \%(\mathrm{H}-1 \rightarrow \mathrm{L}+3), 16 \%(\mathrm{H}-2 \rightarrow \mathrm{L})$ & 4.288 & 289 & & 0.015 \\
\hline
\end{tabular}

${ }^{\mathrm{a}}$ Observed absorption for $\mathbf{1}$ in $\mathrm{CH}_{2} \mathrm{Cl}_{2}$ solution $298 \mathrm{~K}$.

Table SM4. Calculated low-energy singlet excitation energies, wavelengths, and oscillator strengths (OS) for complex [ $\left.\mathrm{Au}\left(2-\mathrm{SC}_{6} \mathrm{H}_{4} \mathrm{NH}_{2}\right)\left(\mathrm{PPh}_{3}\right)\right](\mathbf{1})$

\begin{tabular}{|c|c|c|c|c|}
\hline Composition & $\begin{array}{c}\text { Energy } \\
(\mathrm{eV})\end{array}$ & $\begin{array}{l}\text { Wavelength } \\
(\mathrm{nm})\end{array}$ & $\begin{array}{l}\lambda_{\max }^{a} \\
(\mathrm{~nm})\end{array}$ & $\begin{array}{c}\text { OS } \\
\left(\times 10^{3}\right)\end{array}$ \\
\hline \multicolumn{5}{|l|}{1} \\
\hline $99 \%(\mathrm{H} \rightarrow \mathrm{L})$ & 1.9658 & 630 & & 0.002 \\
\hline $99 \%(\mathrm{H} \rightarrow \mathrm{L}+1)$ & 2.3245 & 533 & & 0.015 \\
\hline $98 \%(\mathrm{H}-1 \rightarrow \mathrm{L}+1)$ & 2.7993 & 443 & & 0.016 \\
\hline $93 \%(\mathrm{H}-2 \rightarrow \mathrm{L}+3)$ & 3.5401 & 350 & & 0.014 \\
\hline $84 \%(H \rightarrow L+6)$ & 3.6473 & 340 & & 0.011 \\
\hline $\begin{array}{l}33 \%(\mathrm{H} \rightarrow \mathrm{L}+7), 21 \%(\mathrm{H} \rightarrow \mathrm{L}+8), \\
13 \%(\mathrm{H}-1 \rightarrow \mathrm{L}+6)\end{array}$ & 4.1682 & 297 & 311 & 0.076 \\
\hline $63 \%(\mathrm{H}-3 \rightarrow \mathrm{L}+1), 12 \%(\mathrm{H}-4 \rightarrow \mathrm{L}+1)$ & 4.3227 & 287 & & 0.026 \\
\hline
\end{tabular}

${ }^{\mathrm{a}}$ Observed absorption for $\mathbf{1}$ in $\mathrm{CH}_{2} \mathrm{Cl}_{2}$ solution $298 \mathrm{~K}$. 
Table SM5. Composition (\%) and energies $(\mathrm{eV})$ of frontier molecular orbitals of $\left[\mathrm{Au}\left(2-\mathrm{SC}_{6} \mathrm{H}_{4} \mathrm{NH}_{2}\right)_{2}\right]^{-}(\mathbf{2})(\mathrm{L}=\mathrm{LUMO}, \mathrm{H}=\mathrm{HOMO})$.

\begin{tabular}{|c|c|c|c|c|c|c|c|c|}
\hline & & & & & Com & sitions ( & & \\
\hline & & Energy & $\mathrm{Au}$ & $\mathrm{S}$ & $S^{\prime}$ & thiolate & thiolate' & Main components \\
\hline $55 a$ & $\mathrm{H}-3$ & -2.125 & 5.4 & 11.6 & 0.2 & 80.9 & 5.7 & $\mathrm{~N}\left(\mathrm{p}_{\mathrm{z}}\right)+\mathrm{C}\left(\mathrm{p}_{\mathrm{z}}\right)$ \\
\hline $56 a$ & $\mathrm{H}-2$ & -1.911 & 9.0 & 11.2 & 28.3 & 11.8 & 73.7 & $S^{\prime}\left(p_{y}\right)$ \\
\hline $57 \mathrm{a}$ & $\mathrm{H}-1$ & -1.634 & 11.9 & 0.9 & 58.5 & 5.6 & 78.0 & $S^{\prime}\left(p_{z}, p_{y}\right)$ \\
\hline $58 \mathbf{a}$ & $\mathbf{H}$ & -1.116 & 10.3 & 48.9 & 3.4 & 79.6 & 4.3 & $\mathrm{~S}\left(\mathrm{p}_{\mathrm{z}}, \mathrm{p}_{\mathrm{x}}\right)$ \\
\hline 59a & $\mathbf{L}$ & 1.680 & 11.5 & 2.5 & 0.2 & 6.9 & 77.2 & $\mathrm{C}_{\text {ring }}^{\prime}\left(\mathrm{p}_{\mathrm{x}}\right)$ \\
\hline $60 \mathrm{a}$ & $\mathrm{L}+1$ & 1.935 & 1.9 & 1.7 & 0.2 & 73.4 & 15.2 & $\mathrm{C}_{\text {ring }}\left(\mathrm{p}_{\mathrm{z}}\right)$ \\
\hline $61 \mathrm{a}$ & $\mathrm{L}+2$ & 2.098 & 3.0 & 1.8 & 0.0 & 26.2 & 67.3 & $C_{\text {ring }}^{\prime}\left(p_{x}\right)$ \\
\hline $62 \mathrm{a}$ & $\mathrm{L}+3$ & 2.187 & 5.7 & 3.0 & 2.4 & 36.9 & 58.1 & $\mathrm{C}_{\text {ring }}^{\prime}\left(\mathrm{p}_{\mathrm{x}}, \mathrm{p}_{\mathrm{z}}\right)$ \\
\hline
\end{tabular}

Table SM6. Calculated low-energy singlet excitation energies, wavelengths, and oscillator strengths $(\mathrm{OS})$ of $\left[\mathrm{Au}\left(2-\mathrm{SC}_{6} \mathrm{H}_{4} \mathrm{NH}_{2}\right)_{2}\right]^{-}$(2).

\begin{tabular}{lcccc}
\hline \multicolumn{1}{c}{ Composition } & $\begin{array}{c}\text { Energy } \\
(\mathrm{eV})\end{array}$ & $\begin{array}{c}\text { Wavelength } \\
(\mathrm{nm})\end{array}$ & $\begin{array}{c}\lambda_{\max }{ }^{\mathrm{a}} \\
(\mathrm{nm})\end{array}$ & $\begin{array}{c}\text { OS } \\
\left(\mathrm{x} 10^{3}\right)\end{array}$ \\
\hline $55 \%(\mathrm{H} \rightarrow \mathrm{L}+1), 38 \%(\mathrm{H} \rightarrow \mathrm{L}+2)$ & 3.1618 & 392 & & 0.018 \\
$87 \%(\mathrm{H}-1 \rightarrow \mathrm{L}), 10 \%(\mathrm{H}-2 \rightarrow \mathrm{L})$ & 3.3929 & 365 & 319 & 0.020 \\
$32 \%(\mathrm{H} \rightarrow \mathrm{L}+3), 22 \%(\mathrm{H} \rightarrow \mathrm{L}+1)$, & 3.4502 & 359 & & \\
$19 \%(\mathrm{H} \rightarrow \mathrm{L}+2)$ & & & & 0.018 \\
$83 \%(\mathrm{H}-1 \rightarrow \mathrm{L}+1)$ & 3.6122 & 343 & & 0.012 \\
$39 \%(\mathrm{H}-1 \rightarrow \mathrm{L}+2), 26 \%(\mathrm{H}-2 \rightarrow \mathrm{L})$, & 3.7596 & 330 & & 0.018 \\
$17 \%(\mathrm{H}-3 \rightarrow \mathrm{L})$ & & & & 0.021 \\
$87 \%(\mathrm{H} \rightarrow \mathrm{L}+5), 10 \%(\mathrm{H}-3 \rightarrow \mathrm{L})$ & 3.8041 & 326 & & \\
$36 \%(\mathrm{H}-1 \rightarrow \mathrm{L}+3), 23 \%(\mathrm{H}-2 \rightarrow \mathrm{L}+1)$, & 3.8386 & 323 & & \\
$17 \%(\mathrm{H} \rightarrow \mathrm{L}+4)$ & & & &
\end{tabular}

${ }^{\mathrm{a}}$ Observed absorption for 2 in $\mathrm{CH}_{2} \mathrm{Cl}_{2}$ solution $298 \mathrm{~K}$.

Table SM7. Composition (\%) and energies $(\mathrm{eV})$ of frontier molecular orbitals of two models $\mathbf{3 h}$ and $\mathbf{3 m}$ of $\left[\left\{\mathrm{Au}\left(2-\mathrm{SC}_{6} \mathrm{H}_{4} \mathrm{NH}_{2}\right)\right\}_{2}(\mu\right.$-dppm) $]$ (3) (L = LUMO, $\left.\mathrm{H}=\mathrm{HOMO}\right)$.

\begin{tabular}{|c|c|c|c|c|c|c|c|}
\hline \multicolumn{8}{|c|}{ Compositions (\%) } \\
\hline \multicolumn{2}{|c|}{ MO } & Energy (eV) & $2 \mathrm{Au}$ & $2 \mathrm{~S}$ & 2 thiolate & phosphine & Main components of orbital \\
\hline \multicolumn{8}{|r|}{ 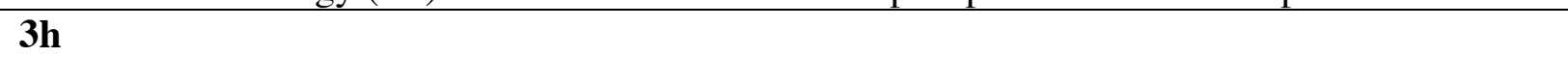 } \\
\hline $80 \mathrm{a}$ & H-4 & -5.067 & 11.3 & 39.8 & 79.6 & 1.2 & $\mathrm{~S}\left(\mathrm{p}_{\mathrm{z}}\right)$ \\
\hline $81 \mathrm{a}$ & H-3 & -4.911 & 2.9 & 52.1 & 89.0 & 1.3 & $\mathrm{~S}\left(\mathrm{p}_{\mathrm{y}}, \mathrm{p}_{\mathrm{x}}\right)$ \\
\hline $82 \mathrm{a}$ & $\mathrm{H}-2$ & -4.833 & 1.9 & 43.0 & 89.4 & 1.0 & $\mathrm{~S}\left(\mathrm{p}_{\mathrm{z}}\right)$ \\
\hline $83 a$ & $\mathrm{H}-1$ & -4.412 & 5.1 & 20.5 & 86.8 & 1.2 & $S\left(p_{y}\right)+N\left(p_{y}\right)$ \\
\hline $84 a$ & $\mathbf{H}$ & -4.161 & 3.9 & 29.1 & 89.2 & 0.8 & $\mathrm{~S}\left(\mathrm{p}_{\mathrm{y}}\right)+\mathrm{C}_{\text {thiolate }}\left(\mathrm{p}_{\mathrm{y}}\right)+\mathrm{N}\left(\mathrm{p}_{\mathrm{y}}\right)$ \\
\hline 85a & $\mathbf{L}$ & -2.795 & 16.6 & 3.8 & 6.1 & 72.9 & $P\left(p_{x}, p_{y}\right)$ \\
\hline $86 a$ & $\mathrm{~L}+1$ & -2.241 & 12.6 & 3.1 & 3.6 & 81.4 & $P\left(p_{z}, p_{x}\right)$ \\
\hline $87 \mathrm{a}$ & $\mathrm{L}+2$ & -1.807 & 18.3 & 1.8 & 4.8 & 72.2 & $\mathrm{P}\left(\mathrm{p}_{\mathrm{z}}, \mathrm{p}_{\mathrm{x}}\right)$ \\
\hline \multicolumn{8}{|l|}{$3 \mathrm{~m}$} \\
\hline $92 a$ & H-4 & -5.115 & 22.1 & 12.8 & 53.4 & 1.5 & $S\left(p_{z}\right)+A u(s)$ \\
\hline $93 a$ & H-3 & -4.772 & 4.9 & 32.8 & 82.0 & 1.5 & $\mathrm{~S}\left(\mathrm{p}_{\mathrm{z}}\right)+\mathrm{C}\left(\mathrm{p}_{\mathrm{y}}\right)$ \\
\hline
\end{tabular}




\begin{tabular}{cccccccc}
$94 \mathrm{a}$ & $\mathrm{H}-2$ & -4.695 & 6.0 & 25.9 & 63.3 & 1.8 & $\mathrm{~S}\left(\mathrm{p}_{\mathrm{z}}, \mathrm{p}_{\mathrm{y}}\right)$ \\
$95 \mathrm{a}$ & $\mathrm{H}-1$ & -4.304 & 4.7 & 18.6 & 84.9 & $\mathbf{1 . 6}$ & $\mathrm{S}\left(\mathrm{p}_{\mathrm{y}}\right)+\mathrm{N}\left(\mathrm{p}_{\mathrm{y}}\right)$ \\
$\mathbf{9 6 a}$ & $\mathbf{H}$ & $\mathbf{- 4 . 0 8 3}$ & $\mathbf{4 . 5}$ & $\mathbf{3 2 . 3}$ & $\mathbf{8 7 . 9}$ & $\mathbf{1 . 4}$ & $\mathrm{S}\left(\mathrm{p}_{\mathrm{y}}, \mathrm{p}_{\mathrm{z}}\right)+\mathrm{C}_{\text {thiolate }}\left(\mathrm{p}_{\mathrm{y}}\right)+\mathrm{N}\left(\mathrm{p}_{\mathrm{y}}\right)$ \\
$\mathbf{9 7 a}$ & $\mathbf{L}$ & $\mathbf{- 1 . 6 2 5}$ & $\mathbf{3 4 . 4}$ & $\mathbf{2 . 6}$ & $\mathbf{1 0 . 9}$ & 47.4 & $\mathrm{P}\left(\mathrm{p}_{\mathrm{x}}\right)+\mathrm{Au}\left(\mathrm{p}_{\mathrm{x}}\right)$ \\
$98 \mathrm{a}$ & $\mathrm{L}+1$ & -1.445 & 23.2 & 3.6 & 8.3 & 64.4 & $\mathrm{P}\left(\mathrm{p}_{\mathrm{z}}\right)+\mathrm{Au}\left(\mathrm{p}_{\mathrm{z}}\right)$ \\
$99 \mathrm{a}$ & $\mathrm{L}+2$ & -1.010 & 7.5 & 2.1 & 7.7 & 82.0 & $\mathrm{H}_{\mathrm{Me}}(\mathrm{s})+\mathrm{P}(\mathrm{s})$ \\
\hline
\end{tabular}

Table SM8. Calculated low-energy singlet excitation energies, wavelengths, and oscillator strengths $(\mathrm{OS})$ for the model $\mathbf{3 h}$ of $\left[\left\{\mathrm{Au}\left(2-\mathrm{SC}_{6} \mathrm{H}_{4} \mathrm{NH}_{2}\right)\right\}_{2}(\mu\right.$-dppm) $](\mathbf{3})$.

\begin{tabular}{lcccc}
\hline \multicolumn{1}{c}{ Composition } & $\begin{array}{c}\text { Energy } \\
(\mathrm{eV})\end{array}$ & $\begin{array}{c}\text { Wavelength } \\
(\mathrm{nm})\end{array}$ & $\begin{array}{c}\lambda_{\max }{ }^{\mathrm{a}} \\
(\mathrm{nm})\end{array}$ & $\begin{array}{c}\text { OS } \\
\left(\mathrm{x} 10^{3}\right)\end{array}$ \\
\hline \multicolumn{1}{c}{ 3h } & & & & \\
\hline $97 \%(\mathrm{H}-1 \rightarrow \mathrm{L})$ & 1.697 & 730 & 0.044 \\
$72 \%(\mathrm{H}-1 \rightarrow \mathrm{L}+1), 21 \%(\mathrm{H}-3 \rightarrow \mathrm{L})$ & 2.206 & 562 & 0.018 \\
$94 \%(\mathrm{H}-4 \rightarrow \mathrm{L})$ & 2.324 & 533 & 0.034 \\
$93 \%(\mathrm{H} \rightarrow \mathrm{L}+2)$ & 2.389 & 519 & 0.022 \\
$70 \%(\mathrm{H}-1 \rightarrow \mathrm{L}+2), 19 \%(\mathrm{H}-2 \rightarrow \mathrm{L}+1)$ & 2.628 & 498 & 0.018 \\
$50 \%(\mathrm{H}-3 \rightarrow \mathrm{L}+1), 31 \%(\mathrm{H}-5 \rightarrow \mathrm{L})$ & 2.676 & 463 & 0.014 \\
$42 \%(\mathrm{H}-3 \rightarrow \mathrm{L}+1), 25 \%(\mathrm{H}-5 \rightarrow \mathrm{L})$ & 2.754 & 450 & 0.044 \\
$79 \%(\mathrm{H} \rightarrow \mathrm{L}+1)$ & 2.865 & 433 & 0.022 \\
$92 \%(\mathrm{H}-1 \rightarrow \mathrm{L}+4)$ & 2.904 & 427 & 0.016 \\
$93 \%(\mathrm{H} \rightarrow \mathrm{L}+5)$ & 3.097 & 400 & 0.034 \\
$78 \%(\mathrm{H}-5 \rightarrow \mathrm{L}+1)$ & 3.262 & 380 & 0.038 \\
$42 \%(\mathrm{H}-2 \rightarrow \mathrm{L}+4), 29 \%(\mathrm{H}-4 \rightarrow \mathrm{L}+2)$ & 3.294 & 376 & 0.034 \\
$42 \%(\mathrm{H}-2 \rightarrow \mathrm{L}+4), 23 \%(\mathrm{H}-4 \rightarrow \mathrm{L}+2)$ & 3.310 & 374 & & \\
\hline
\end{tabular}

Table SM9. Calculated low-energy singlet excitation energies, wavelengths, and oscillator strengths (OS) for the model $\mathbf{3 m}$ of $\left[\left\{\mathrm{Au}\left(2-\mathrm{SC}_{6} \mathrm{H}_{4} \mathrm{NH}_{2}\right)\right\}_{2}(\mu\right.$-dppm) $](3)$.

\begin{tabular}{lcccc}
\hline \multicolumn{1}{c}{ Composition } & $\begin{array}{c}\text { Energy } \\
(\mathrm{eV})\end{array}$ & $\begin{array}{c}\text { Wavelength } \\
(\mathrm{nm})\end{array}$ & $\begin{array}{c}\lambda_{\max }{ }^{\mathrm{a}} \\
(\mathrm{nm})\end{array}$ & $\begin{array}{c}\text { OS } \\
\left(\mathrm{x} 10^{3}\right)\end{array}$ \\
\hline $96 \%(\mathrm{H} \rightarrow \mathrm{L}+1)$ & 2.721 & 456 & & 0.024 \\
$97 \%(\mathrm{H}-1 \rightarrow \mathrm{L})$ & 2.741 & 452 & & 0.036 \\
$61 \%(\mathrm{H} \rightarrow \mathrm{L}+3), 20 \%(\mathrm{H} \rightarrow \mathrm{L}+2)$ & 3.161 & 392 & & 0.026 \\
$38 \%(\mathrm{H} \rightarrow \mathrm{L}+4,34 \%(\mathrm{H}-2 \rightarrow \mathrm{L}+1)$, & 3.314 & 374 & & 0.024 \\
$34 \%(\mathrm{H}-1 \rightarrow \mathrm{L}+2)$ & & & & \\
$55 \%(\mathrm{H} \rightarrow \mathrm{L}+5), 22 \%(\mathrm{H}-3 \rightarrow \mathrm{L}+1)$ & 3.491 & 355 & & 0.064 \\
$79 \%(\mathrm{H}-1 \rightarrow \mathrm{L}+4)$ & 3.564 & 348 & & 0.020 \\
$83 \%(\mathrm{H}-4 \rightarrow \mathrm{L})$ & 3.591 & 345 & & 0.080 \\
$53 \%(\mathrm{H} \rightarrow \mathrm{L}+6), 21 \%(\mathrm{H}-1 \rightarrow \mathrm{L}+5)$ & 3.624 & 342 & & 0.026 \\
$59 \%(\mathrm{H} \rightarrow \mathrm{L}+6)$ & 3.660 & 339 & & 0.023 \\
$76 \%(\mathrm{H}-2 \rightarrow \mathrm{L}+3)$ & 3.746 & 331 & & 0.018 \\
$76 \%(\mathrm{H}-5 \rightarrow \mathrm{L})$ & 3.851 & 322 & 308 & 0.092 \\
\hline
\end{tabular}

${ }^{\mathrm{a}}$ Observed absorption for $\mathbf{3}$ in $\mathrm{CH}_{2} \mathrm{Cl}_{2}$ solution $298 \mathrm{~K}$. 
Table SM10. Composition (\%) and energies (eV) of frontier molecular orbitals of the model $4 \mathbf{h}$ of $\left[\left(\mathrm{AuPPh}_{3}\right)\left\{\mathrm{Au}\left(\mathrm{C}_{6} \mathrm{~F}_{5}\right)_{3}\right\}\left(\mu_{2}-2-\mathrm{SC}_{6} \mathrm{H}_{4} \mathrm{NH}_{2}\right)\right](\mathbf{4})(\mathrm{L}=\mathrm{LUMO}, \mathrm{H}=\mathrm{HOMO})$.

$$
\text { Compositions (\%) }
$$

MO Energy $(\mathrm{eV}) \mathrm{Au} \quad \mathrm{S}$ thiolate phosphine $A u \quad A u\left(C F_{3}\right)_{3}$ components of orbital

\begin{tabular}{cccccccccc}
\hline 4h & & & & & & & \\
\hline $92 \mathrm{a}$ & H-3 & -7.251 & 26.3 & 34.8 & 49.4 & 0.6 & 11.8 & 18.7 & $\mathrm{~S}\left(\mathrm{p}_{\mathrm{z}}\right)+\mathrm{Au}(\mathrm{s})$ \\
$93 \mathrm{a}$ & H-2 & -6.501 & 0.2 & 0.7 & 1.4 & 0.0 & 11.9 & 92.8 & $\mathrm{C}(\mathrm{s})+A u\left(\mathrm{p}_{\mathrm{x}}\right)$ \\
$94 \mathrm{a}$ & H-1 & -6.188 & 1.8 & 16.3 & 82.4 & 0.4 & 3.0 & 10.3 & $\mathrm{C}_{\text {thiolate }}\left(\mathrm{p}_{\mathrm{y}}\right)$ \\
$\mathbf{9 5 a}$ & $\mathbf{H}$ & -5.297 & $\mathbf{0 . 3}$ & $\mathbf{2 . 8}$ & $\mathbf{8 9 . 4}$ & $\mathbf{0 . 1}$ & $\mathbf{1 . 4}$ & $\mathbf{3 . 8}$ & $\mathrm{N}\left(\mathrm{p}_{\mathrm{y}}\right)+\mathrm{C}_{\text {thiolate }}\left(\mathrm{p}_{\mathrm{y}}\right)$ \\
$\mathbf{9 6 a}$ & $\mathbf{L}$ & -2.843 & $\mathbf{2 . 6}$ & $\mathbf{9 . 6}$ & $\mathbf{1 8 . 5}$ & $\mathbf{5 . 1}$ & $\mathbf{2 6 . 4}$ & $\mathbf{6 9 . 1}$ & $\mathrm{Au}\left(\mathrm{d}_{\mathrm{xy}}\right)$ \\
$97 \mathrm{a}$ & L+1 & -2.579 & 25.0 & 2.7 & 6.7 & 56.6 & 4.1 & 6.1 & $\mathrm{P}\left(\mathrm{p}_{\mathrm{x}}\right)+\mathrm{Au}\left(\mathrm{p}_{\mathrm{x}}\right)$ \\
$98 \mathrm{a}$ & L+2 & -2.439 & 19.0 & 3.2 & 12.1 & 62.7 & 2.9 & 6.0 & $\mathrm{P}\left(\mathrm{p}_{\mathrm{y}}\right)+\mathrm{Au}\left(\mathrm{p}_{\mathrm{y}}\right)$ \\
$99 \mathrm{a}$ & L+3 & -2.289 & 47.2 & 8.2 & 9.9 & 40.0 & 0 & 0.4 & $\mathrm{Au}(\mathrm{s})+\mathrm{P}(\mathrm{s})$ \\
\hline
\end{tabular}

Table SM11. Calculated low-energy singlet excitation energies, wavelengths, and oscillator strengths $(\mathrm{OS})$ of the model $\mathbf{4 h}$ of $\left[\left(\mathrm{AuPPh}_{3}\right)\left\{\mathrm{Au}\left(\mathrm{C}_{6} \mathrm{~F}_{5}\right)_{3}\right\}\left(\mu_{2}-2-\mathrm{SC}_{6} \mathrm{H}_{4} \mathrm{NH}_{2}\right)\right]$ (4).

\begin{tabular}{lcccc}
\hline \multicolumn{1}{c}{ Composition } & $\begin{array}{c}\text { Energy } \\
(\mathrm{eV})\end{array}$ & $\begin{array}{c}\text { Wavelength } \\
(\mathrm{nm})\end{array}$ & $\begin{array}{c}\lambda_{\max }{ }^{\mathrm{a}} \\
(\mathrm{nm})\end{array}$ & $\begin{array}{c}\text { OS } \\
\left(\mathrm{x} 10^{3}\right)\end{array}$ \\
\hline $92 \%(\mathrm{H} \rightarrow \mathrm{L})$ & 2.5898 & 479 & & 0.030 \\
$91 \%(\mathrm{H} \rightarrow \mathrm{L}+2)$ & 2.9256 & 424 & & 0.035 \\
$98 \%(\mathrm{H} \rightarrow \mathrm{L}+3)$ & 3.0224 & 410 & & 0.003 \\
$61 \%(\mathrm{H}-1 \rightarrow \mathrm{L}), 27 \%(\mathrm{H}-1 \rightarrow \mathrm{L}+1)$, & 3.5890 & 345 & 345 & 0.074 \\
$70 \%(\mathrm{H}-1 \rightarrow \mathrm{L}+1), 17 \%(\mathrm{H}-1 \rightarrow \mathrm{L})$ & 3.6431 & 340 & & 0.023 \\
$75 \%(\mathrm{H}-1 \rightarrow \mathrm{L}+2), 11 \%(\mathrm{H}-1 \rightarrow \mathrm{L})$ & 3.8753 & 319 & & 0.073 \\
$60 \%(\mathrm{H}-2 \rightarrow \mathrm{L}+2), 37 \%(\mathrm{H}-2 \rightarrow \mathrm{L})$ & 4.0239 & 308 & & 0.018 \\
\hline
\end{tabular}

${ }^{\mathrm{a}}$ Observed absorption for 4 in $\mathrm{CH}_{2} \mathrm{Cl}_{2}$ solution $298 \mathrm{~K}$.

Table SM12. Composition (\%) and energies (eV) of frontier molecular orbitals of the model $\mathbf{5 h}$ of $\left[\left(\mathrm{C}_{6} \mathrm{~F}_{5}\right)_{3} \mathrm{Au}\left(\mu_{2}-2-\mathrm{SC}_{6} \mathrm{H}_{4} \mathrm{NH}_{2}\right)(\mathrm{AudppmAu})\left(\mu_{2}-2-\mathrm{SC}_{6} \mathrm{H}_{4} \mathrm{NH}_{2}\right) \mathrm{Au}\left(\mathrm{C}_{6} \mathrm{~F}_{5}\right)_{3}\right]$ (5) ( $\mathrm{L}=\mathrm{LUMO}, \mathrm{H}=\mathrm{HOMO})$.

\begin{tabular}{|c|c|c|c|c|c|c|c|c|c|}
\hline \multicolumn{10}{|c|}{ Compositions (\%) } \\
\hline \multicolumn{2}{|c|}{ MO } & Energy $(\mathrm{eV})$ & $\mathrm{Au}$ & $\mathrm{S}$ & thiolate & phosphine & $A u$ & $A u\left(\mathrm{CF}_{3}\right)_{3}$ & components of orbital \\
\hline $5 \mathrm{~h}$ & & & & & & & & & \\
\hline $189 \mathrm{a}$ & H-3 & -6.294 & 1.6 & 14.5 & 81.8 & 0.6 & 2.6 & 9.5 & $\mathrm{C}_{\text {thiolate }}\left(\mathrm{p}_{\mathrm{x}}\right)$ \\
\hline $190 \mathrm{a}$ & $\mathrm{H}-2$ & -6.225 & 2.9 & 16.2 & 78.7 & 0.3 & 3.3 & 11.0 & $\mathrm{C}_{\text {thiolate }}\left(\mathrm{p}_{\mathrm{x}}\right)$ \\
\hline $191 \mathrm{a}$ & $\mathrm{H}-1$ & -5.393 & 0.5 & 2.2 & 89.3 & 0.3 & 1.1 & 2.9 & $\mathrm{~N}\left(\mathrm{p}_{\mathrm{x}}\right)+\mathrm{C}_{\text {thiolate }}\left(\mathrm{p}_{\mathrm{x}}\right)$ \\
\hline $192 a$ & H & -5.355 & 0.5 & 2.8 & 88.0 & 0.1 & 1.5 & 3.9 & $\mathrm{~N}\left(\mathrm{p}_{\mathrm{x}}\right)+\mathrm{C}_{\text {thiolate }}\left(\mathrm{p}_{\mathrm{x}}\right)$ \\
\hline 193a & $\mathbf{L}$ & -3.672 & 25.8 & 2.7 & 3.6 & 57.7 & 2.3 & 4.4 & $P\left(p_{x}\right)+A u\left(p_{x}\right)$ \\
\hline $194 a$ & $\mathrm{~L}+1$ & -3.102 & 15.2 & 1.0 & 2.5 & 55.4 & 7.1 & 17.3 & $P\left(p_{z}\right)+A u\left(p_{z}\right)$ \\
\hline $195 \mathrm{a}$ & $\mathrm{L}+2$ & -2.996 & 26.8 & 4.4 & 6.6 & 53.7 & 1.9 & 4.9 & $P\left(p_{y}, p_{z}\right)+A u\left(p_{y}, p_{z}\right)$ \\
\hline $196 \mathrm{a}$ & $\mathrm{L}+3$ & -2.810 & 4.0 & 8.2 & 22.1 & 11.5 & 22.0 & 53.2 & $\mathrm{~S}\left(\mathrm{p}_{\mathrm{x}}\right)+A u\left(\mathrm{~d}_{\mathrm{z}}^{2}\right)$ \\
\hline
\end{tabular}


Table SM13. Calculated low-energy singlet excitation energies, wavelengths, and oscillator strengths (OS) of the model $\mathbf{5 h}$ of complex $\left[\left(\mathrm{C}_{6} \mathrm{~F}_{5}\right)_{3} \mathrm{Au}\left(\mu_{2}-2-\mathrm{SC}_{6} \mathrm{H}_{4} \mathrm{NH}_{2}\right)(\mathrm{AudppmAu})\left(\mu_{2}-2-\mathrm{SC}_{6} \mathrm{H}_{4} \mathrm{NH}_{2}\right) \mathrm{Au}\left(\mathrm{C}_{6} \mathrm{~F}_{5}\right)_{3}\right]$ (5). (only the strongest excitations are shown)

\begin{tabular}{lcccc}
\hline \multicolumn{1}{c}{ Composition } & $\begin{array}{c}\text { Energy } \\
(\mathrm{eV})\end{array}$ & $\begin{array}{c}\text { Wavelength } \\
(\mathrm{nm})\end{array}$ & $\begin{array}{c}\lambda_{\max }^{\mathrm{a}} \\
(\mathrm{nm})\end{array}$ & $\begin{array}{c}\text { OS } \\
\left(\mathrm{x} 10^{3}\right)\end{array}$ \\
\hline $100 \%(\mathrm{H} \rightarrow \mathrm{L})$ & 1.6977 & 730 & & 0.009 \\
$96 \%(\mathrm{H}-1 \rightarrow \mathrm{L})$ & 2.5759 & 481 & & 0.017 \\
$78 \%(\mathrm{H}-1 \rightarrow \mathrm{L}+3), 10 \%(\mathrm{H} \rightarrow \mathrm{L}+3)$ & 2.7304 & 454 & & 0.033 \\
$61 \%(\mathrm{H} \rightarrow \mathrm{L}+4), 16 \%(\mathrm{H}-1 \rightarrow \mathrm{L}+5)$, & 2.7618 & 448 & 342 & 0.038 \\
$14 \%(\mathrm{H} \rightarrow \mathrm{L}+6)$ & & & & \\
\hline
\end{tabular}

${ }^{\mathrm{a}}$ Observed absorption for $\mathbf{5}$ in $\mathrm{CH}_{2} \mathrm{Cl}_{2}$ solution $298 \mathrm{~K}$.

Table SM14. Relevant calculated distances $(\AA)$ and angles $\left(^{\circ}\right)$ for models of complexes $\mathbf{4 h}$ and $\mathbf{5 h}$, compared with experimental values for $\mathbf{5}$.

\begin{tabular}{cccc}
\hline Distance/angle & $\mathbf{5}$ & $\mathbf{5 h}$ & $\mathbf{4 h}$ \\
\hline $\mathrm{Au} . . \mathrm{Au}$ & 2.935 & 3.294 & - \\
$\mathrm{Au}-\mathrm{S}$ & $2.332,2.341$ & $2.243,2.336$ & 2.343 \\
$\mathrm{Au}-\mathrm{P}$ & $2.270,2.269$ & $2.268,2.270$ & 2.266 \\
$\mathrm{P}-\mathrm{C}$ & $1.832,1.880$ & $1.859,1.860$ & \\
$A u-\mathrm{S}^{*}$ & $2.372,2.375$ & $2.483,2.478$ & 2.502 \\
$\mathrm{Au}-\mathrm{Au}$ & $3.281,3.382$ & $3.689,3.779$ & - \\
$\mathrm{S}-\mathrm{Au}-\mathrm{P}$ & $176.2,174.5$ & $171.2,174.3$ & 177.2 \\
$\mathrm{Au}-\mathrm{S}-\mathrm{Au}$ & $91.9,88.2$ & $99.7,103.4$ & 93.1 \\
$\mathrm{C}-\mathrm{S}-\mathrm{Au}$ & $107.8,109.2$ & $110.3,110.3$ & 109.1 \\
$\mathrm{Au}-\mathrm{P} . \mathrm{P}-\mathrm{Au}$ & 35.4 & 50.1 & -
\end{tabular}

$* \mathrm{Au}(\mathrm{III})=A \bar{u}$ 